\title{
La relación entre la irritación laboral y la adicción al trabajo en una muestra española multiocupacional*
}

\section{The Relationship between Irritation at Work and Workaholism in a Spanish Multi-Occupational Sample}

Recibido: septiembre 22 de 2012 | Revisado: mayo 26 de 2013 | Aceptado: junio 18 de 2013

\author{
ENRIQUe MERINO-TEJEDOR ** \\ Universidad de Valladolid, España \\ JOAN BOADA-GRAU *** \\ Universitat Rovira i Virgili, Tarragona, España \\ Aldo Javier Prizmic-KuZMiCA ***** \\ Escuela de Alta Dirección y Administración,
}

Barcelona, España

doi:10.11144/Javeriana.UPSY13-2.rila

Para citar este artículo: Merino-Tejedor, E., Boada-Grau, J. \& Prizmic-Kuzmica, A. J. (2014). La relación entre la irritación laboral y la adicción al trabajo en una muestra española multiocupacional. Universitas Psychologica, 13(2), 477-489. doi:10.11144/ Javeriana.UPSY13-2.rila

* Artículo de investigación.

** Correspondencia Universidad de Valladolid, Departamento de Psicología, Facultad de Ciencias Sociales. Casa de la Tierra. Plaza de la Tierra, 3. 40.001 Segovia, España. Tel. +34 921 112100. Fax +34921 112101.E-mail: enmerino@psi.uva.es

**** Universitat Rovira i Virgili, Facultat de Ciencias de la Educación i Psicología. Campus Sescelades. Carretera de Valls, s/n. 43007 Tarragona, España. Tel: (34) 977.55.81.61.E-mail: joan.boada@urv.cat

******* Escuela de Alta Dirección y Administración. Aragón, 204. 08011 Barcelona. Tel: (34) 934.520.844.E-mail: jprizmic@eada.edu

\section{RESUMEN}

El trabajo tiene connotaciones positivas, pero también puede ser un escenario donde se presentan riesgos que pueden desarrollar aspectos negativos. Esta investigación intenta profundizar en la relación existente entre dos conceptos del escenario laboral: la irritación laboral con sus dos dimensiones, irritación cognitiva y emocional, y la adicción al trabajo. Hasta la fecha, se han llevado a cabo investigaciones sobre cada uno de estos conceptos en su relación con otras variables, pero no hay estudios que hayan indagado en la relación directa existente entre ambos constructos. Para llevar a cabo la investigación, se pasaron los instrumentos de medida a una muestra de 285 sujetos de diversas ocupaciones y se encontraron resultados en la línea esperada, es decir, una relación positiva y significativa entre la irritación laboral y la adicción al trabajo. Las correlaciones fueron significativas, tanto en el caso de la irritación emocional como de la cognitiva, con la adicción al trabajo medida a través del Dutch Work Addiction Scale. Sin embargo, en el caso del WorkBAT, la irritación solo obtuvo correlaciones significativas en el caso del Factor 1, trabajar excesivamente. En cuanto al análisis de regresión, se obtuvieron resultados interesantes para entender la relación entre ambos conceptos.

Palabras clave

Irritación cognitiva, irritación emocional, adicción al trabajo.

\section{A B S T R ACT}

Work offers a positive side but also it can be a framework where some risks may appear that evolve in negative outcomes. This research focus on the possible relation between two aspects of the work context: irritation at work with its two dimensions, cognitive and emotional irritation, and workaholism. So far the two concepts have been studied in their relation to other variables, but there is a lack of studies on the relation between them. To carry out this investigation 285 subjects from different occupations answered the instruments used and we found results in the expected way. Irritation, both cognitive and emotional irritation, showed significant correlations with both factors of the DUWAS However, in the case of the WBAT the correlations were significant only for factor 1, working excessively. Regression analysis yielded interesting results in order to explain the relation between both concepts. Keywords

Cognitive irritation, emotional irritation, workaholism. 
El trabajo es una faceta muy importante en la vida de las personas. En la actualidad, el trabajo se configura como una actividad de suma trascendencia para los seres humanos, no tanto por lo que supone como medio de supervivencia, sino por configurarse como un medio de realización personal. Por tanto, el trabajo lleva asociadas unas innegables connotaciones positivas, pero, al mismo tiempo, también puede ser un escenario donde se presentan una serie de riesgos que pueden desarrollar aspectos negativos. El presente estudio pretende buscar evidencia empírica en torno a la relación entre dos conceptos importantes del mundo laboral como son la adicción al trabajo (workaholism) y la irritación laboral, dos aspectos muy estudiados dentro de la psicología del trabajo y de las organizaciones. En cuanto a la adicción al trabajo, se trata de un concepto cuyo interés se encuentra tanto dentro de la psicología del trabajo (Porter, 1996) como dentro de otras perspectivas más clínicas de la psicología (Berglas, 2004). Aunque de un modo intuitivo se puede especular con una relación positiva entre ambos conceptos, la verdad es que no existen estudios que se hayan centrado en el interés de este tipo de investigación. Veamos a continuación con más detalle las aportaciones teóricas que se han hecho en torno a cada uno de los conceptos.

\section{La adicción al trabajo}

El reconocimiento de la adicción al trabajo queda puesto de manifiesto en la cantidad de estudios recientes que han aparecido en los últimos años en torno a esta temática (Fassel, 1990; Robinson, 1998; Smith \& Seymour, 2004). Algunos estudios (Burke, Matthiesen \& Pallesen, 2006) han señalado la doble versión que tiene el concepto, ya que algunos autores señalan las consecuencias positivas del concepto para la organización (Machlowitz, 1980; Naughton, 1987; Sprankle \& Ebel, 1987) y otros señalan el lado negativo (Killinger, 1991; Porter, 1996; Schaef \& Fassel, 1988). Aunque la adicción al trabajo puede tener relación con el engagement, algunos estudios han demostrado que se trata de aspectos independientes, encontrando diferencias en su patrón de motivaciones, así mientras los adictos al trabajo se mueven por motivaciones controladas, los trabajadores comprometidos (engagement) lo hacen por motivaciones más autónomas (Van Beek, Taris \& Schaufeli, 2011).

Una de las aportaciones más significativas en torno a la adicción al trabajo vino de la mano de Spence y Robbins (1992). Estos autores formularon un modelo que contempla tres dimensiones importantes: Implicación en el trabajo (work involvement), Disfrute con el trabajo (work enjoyment) e Impulso (drive). Como resultado de sus aportaciones al estudio de este tema, hay que destacar la creación del instrumento conocido como WorkBAT, con las tres dimensiones comentadas. No obstante, hay que señalar que las investigaciones actuales no siempre han confirmado la estructura factorial con tres dimensiones (Kanai, Wakabayashi \& Fling, 1996; McMillan, Brady, O'Driscoll \& Marsh, 2002).

En una excelente investigación actual, $\mathrm{Ng}$, Sorensen y Feldman (2007) hacen una revisión de algunas aproximaciones a la definición de adicción al trabajo, desde la de Oates (1971), que lo concibe como 'una compulsión irrefrenable para trabajar incesantemente', a la de Porter (1996), pasando por la de Fassel (1990) quien lo concibe directamente como 'una patología'. Finalmente, ellos proponen la siguiente definición ( $\mathrm{Ng}$ et al , 2007, p. 114): "los adictos al trabajo son aquellas personas que disfrutan del hecho de trabajar, están obsesionadas con el trabajo, y dedican largas horas y tiempo personal al trabajo". Además, también contemplan tres dimensiones en el concepto de la adicción al trabajo: afectiva, cognitiva y conductual. En cuanto a la dimensión afectiva, se ha hecho una diferenciación entre placer con el trabajo (enjoyment of work) y disfrute al trabajar (enjoyment of working). Además de esta faceta positiva, también estaría la parte negativa de las emociones que vendría de la mano de la culpabilidad y la ansiedad sentidas cuando el trabajador está sin laborar.

Por otro lado, la dimensión cognitiva tendría que ver con pensamientos persistentes sobre el trabajo cuando no se está trabajando (Scott, Moore \& Miceli, 1997), necesidad incontrolable de trabajar (Oates, 1971), un compromiso excesivo e irracional hacia el trabajo (Cherrington, 1980; Porter, 1996) o un impulso interno a trabajar en exceso (Naughton, 
1987; Spence \& Robbins, 1992). Esta dimensión cognitiva de la adicción al trabajo parece que sería la más cercana a una de las dimensiones de la irritación laboral, en concreto la irritación cognitiva, ya que, tal como se describe a continuación, ésta ha sido definida como 'una tendencia a pensar recurrentemente sobre aspectos relacionados con el trabajo y a dar vueltas a los mismos pensamientos'. Por último, la dimensión conductual tendría que ver con una implicación excesiva real dentro del trabajo que un empleado desempeña. Esta implicación se traduciría por ejemplo en dedicar muchas horas al trabajo, y la intrusión de la vida laboral en la vida personal y familiar.

\section{La irritación laboral}

El concepto "irritación" tiene una trayectoria de más de dos décadas, desde su incorporación a la investigación por parte de la investigadora alemana Mohr (1986). En su primera concepción, el concepto estaba vinculado a determinadas experiencias de incertidumbre por parte de los trabajadores. Posteriormente, ese estado de incertidumbre se fue cristalizando en datos más concretos como por ejemplo el hecho de que exista un cierto grado de discrepancia entre una situación laboral actual y los objetivos personales que alguien puede pretender alcanzar. En esta sentido, el concepto de irritación ya estaría relacionado de algún modo con la orientación psicológica que tiende a considerar al trabajador como alguien que busca objetivos (Locke \& Latham, 1990). La percepción del trabajador de obstáculos en su medio para conseguir sus objetivos le produciría cierto nivel de estrés. Según este planteamiento, la irritación sería un resultado de una percepción de discrepancia en la consecución de los objetivos.

Además de esta primera aproximación, el estado de irritación también se asoció a un estado mental de agotamiento psicológico progresivo. En este sentido, era de esperar que los resultados obtenidos con la escala de irritación, mostraran una alta relación con estados similares de estrés como los que se obtienen por ejemplo con el burnout. Las investigaciones experimentales llevadas a cabo así lo han confirmado (Merino, Carbonero, Moreno \&
Morante, 2006). El estudio del estado de irritación tiene un atractivo adicional si se considera que es un concepto concebido como una situación intermedia entre la fatiga psicológica y los desórdenes psicológicos más severos, por tanto la detección precoz de estados de irritación puede ser relevante a la hora de prevenir situaciones más graves.

En los desarrollos empíricos llevados a cabo desde la creación de la escala de irritación (Merino et al., 2006; Mohr, Müller, Rigotti, Aycan \& Tschan, 2006), se ha confirmado la presencia de dos factores claramente identificados dentro del concepto de irritación: la irritación cognitiva y la irritación emocional. El primer componente, también denominado "rumiación", tendría que ver con la tendencia a dar vueltas a los mismos pensamientos de una forma recurrente, sin que sirvan para mejorar la situación. Por otro lado, el segundo componente, también llamado "irritabilidad" tendría que ver con la tendencia a reaccionar de un modo desagradable, incluso hostil, en las situaciones que los sujetos experimenten como estresantes. En opinión de los autores este segundo componente tendría un mayor nivel de severidad para la salud que el primero. La mayor parte de la investigación sobre la irritación se ha centrado en buscar evidencia empírica sobre las dimensiones psicométricas de la escala de irritación, por eso es importante llevar a cabo estudios como el actual que pretenden ampliar el estudio teórico del concepto al relacionarlo con otros constructos, como es el caso de la adicción al trabajo o determinados factores de personalidad.

\section{Relevancia teórica y social}

Por tanto, el presente estudio entraña una importante relevancia y pertinencia teórica, así como una importante repercusión social. Algunos modelos teóricos han apuntado en la dirección de que las personas adictas al trabajo presentan una incapacidad para controlar sus impulsos para trabajar, lo cual les puede llevar a tener problemas sociales o de salud, llegando incluso a experimentar síntomas como la ansiedad cuando se sienten alejados del trabajo (Bakker, Demerouti, Oerlemans \& Sonnentag, 2013; Scott et al., 1997). 
Los hallazgos empíricos encontrados en el presente estudio contribuyen a mejorar los modelos teóricos existentes, ya que, tal como se indicaba previamente, la dimensión cognitiva de la irritación puede estar vinculada con los pensamientos persistentes sobre el trabajo, cuando no se está trabajando (Scott et al., 1997) y con los impulsos internos a trabajar en exceso (Naughton, 1987; Spence \& Robbins, 1992). De este modo, se pueden identificar o mejorar escenarios de prevención e intervención en los entornos laborales, así como desarrollar escalas de mayor precisión y validez que contemplen no solo las dimensiones cognitivas, sino también los aspectos emocionales de la adicción al trabajo, como por ejemplo la culpabilidad y la ansiedad (Ng et al., 2007), la irritabilidad o incluso la propia agresividad (Balducci, Cecchin, Fraccaroli \& Schaufeli, 2012).

Por otro lado y a un nivel de trascendencia social, las transformaciones del empleo, del trabajo y de las organizaciones empresariales, características propias del capitalismo flexible y global, hacen que se tenga que dedicar esfuerzos a entender con mayor profundidad la adicción al trabajo. En este sentido, estudios de revisión actuales ( $\mathrm{Ng}$ et al., 2007) indican la existencia de una serie de factores que pueden estar afectando a una nueva concepción de la adicción al trabajo a nivel social. Tal es el caso de las expectativas de rol claras sobre el trabajo, que dejarán de existir en un futuro cercano (Sullivan, 1999), las propias fronteras entre la vida personal y la vida laboral se están desvaneciendo (Fletcher \& Baylin, 1996) o el surgimiento del teletrabajo gracias a las nuevas tecnologías (Cooper, 1998), que conlleva una nueva concepción de la organización del trabajo a nivel social y personal.

En este contexto, el objetivo principal de este estudio es explorar la relación entre estos dos conceptos: la adicción al trabajo y la irritación laboral. Aunque de modo intuitivo, se puede pensar que existirá una relación positiva entre ambos, es importante analizar exactamente cómo es esa relación y estudiar cómo es la relación también entre las dimensiones de ambos conceptos. Además, como novedad, en este estudio se pretende profundizar en el concepto de irritación, explorando el papel predictivo de la adicción al trabajo mediante un análisis de regresión.

\section{Método}

\section{Participantes}

La muestra multiocupacional utilizada en esta investigación estaba compuesta por 285 trabajadores por cuenta ajena, residentes en España, comunidad autónoma de Cataluña, con una media de edad de 43.31 años $(D E=9.46)$. En cuanto a la distribución por género, el $43.5 \%$ de los participantes eran hombres y el $56.5 \%$ mujeres. En el estado civil la distribución fue la siguiente: $68.9 \%$ casados, $7.8 \%$ convivían en pareja, $7.3 \%$ solteros, $15 \%$ divorciados o separados y $1 \%$ viudos. En cuanto a la formación académica: máster y/o doctorado, 6.7\%; licenciados, $12.9 \%$; diplomados, $14.9 \%$; estudios secundarios, 40.2\%; estudios primarios, $25.3 \%$. En cuanto al sector de actividad productiva, la distribución fue la siguiente: químico (5.4\%), metalúrgico y afines (6.9\%), farmacéutico (5.9\%), alimentación (2.1\%), sanidad y hospitales (10.5\%), telecomunicaciones (7.1\%), informática (3.5\%), turismo (2.4\%), comercio $(7.5 \%)$, hostelería $(2.8 \%)$, mediación financiera (14.7\%), distribución (2.5\%), servicios destinados a ventas (3.6\%), administración pública (6.3\%), educación y servicios sociales (10.6\%), seguridad (3.8\%), consultoría en general (3.3\%) y otros (1.1\%). Por último, la antigüedad media en su trabajo actual fue de 12.11 años $(D E=10.5)$, en su profesión de 18.47 años $(D E=15.54)$ y en su empresa actual de 15.04 años $(D E=11.01)$.

\section{Instrumentos}

En este estudio se utilizaron los tres instrumentos de medida, que se describen a continuación. 
Escala WorkBAT ([WBAT]; Burke, 1999, 2001; Burke, Richardsen Eु Martinussen, 2002; McMillan et al., 2002; Spence EO Robbins, 1992).

Se trata de una escala que evalúa adicción al trabajo, en su versión original en inglés, consta de 25 ítems y tres factores. El primero denominado Implicación en el Trabajo (Work Involvement) con 8 ítems (alpha de Cronbach $=0.67$ ), por ejemplo: "Me aburro y me siento inquieto durante las vacaciones cuando no tengo nada productivo que hacer". El segundo factor es la motivación (Drive), integrado por 7 ítems (alpha de Cronbach $=0.8$ ), tiene que ver con el grado de implicación, compromiso y obligación con el trabajo, así como con la culpabilidad y el aburrimiento cuando no se trabaja, por ejemplo: "Me siento culpable cuando falto al trabajo". Por último, el tercer factor se denomina disfrute del trabajo (Work Enjoyment) formado por 10 ítems (alpha de Cronbach $=0.88$ ), tiene que ver con el hecho de disfrutar trabajando, pasarlo bien, divertirse, por ejemplo: "Mi trabajo es tan interesante que a menudo no parece trabajo".

En cuanto a la versión española (Boada-Grau, Prizmic-Kuzmica, Serrano-Fernández \& VigilColet, en prensa) tiene solo 19 ítems y dos factores. El primer factor incluye a los dos primeros de la versión en inglés y se denomina motivación $(D$, Driven) (alfa $=0.82)$ y tiene 12 ítems, un ejemplo de ítem sería: "A menudo desearía no estar tan comprometido con mi trabajo". El segundo factor, se llama disfrute del trabajo (J, Work Enjoyment) (alfa $=0.83)$ y está configurado por 7 ítems (por ejemplo, "A veces disfruto tanto de mi trabajo que tengo dificultades para dejarlo"). El formato de respuesta es de un rango de cinco puntos en una escala tipo Likert que van desde 1 (nada de acuerdo) hasta 5 (totalmente de acuerdo).

En cuanto a la validez, las dos subescalas del presente instrumento se asocian de forma significativa a otras escalas de contraste. El factor motivación (D, Driven) se asocia al perfeccionismo $(r=0.29, p$ $<0.01$ ). También aparecen correlaciones similares con el MBI-GS (Salanova, Schaufeli, Llorens, Peiró $\&$ Grau, 2000): el agotamiento con D $(r=0.31, p$ $<0.01)$ y con J $(r=-0.21, p<0.01)$, el cinismo con
$\mathrm{J}(r=-0.16, p<0.05)$ y la eficacia personal con D $(r$ $=0.24, p<0.01)$ y J $(r=0.25, p<0.01)$. Además, se indica la vinculación de la adicción del trabajo con la extraversión (con J; $r=0.24, p<0.01$ ), la estabilidad emocional (con J; $r=0.19, p<0.01$ ) y la responsabilidad (con D; $r=0.14, p<0.05$ ).

\section{La Escala de Irritación (Irritation}

Scale) ([IS]; Mohr, 1986)

Esta escala fue diseñada con la finalidad de evaluar el estrés en el trabajo. Este instrumento ha sido validado para evaluar el estrés laboral en una gran variedad de muestras ocupacionales y en diversos países. La Escala de Irritación consta de 8 ítems agrupados en dos factores: un primer factor denominado irritación cognitiva (ejemplo de ítem: "Me resulta difícil desconectar después del trabajo") y un segundo factor llamado irritación emocional (ejemplo de ítem: "Me enfado con facilidad"). Los ítems se valoran en una escala tipo Likert que van desde 1 (muy en desacuerdo) a 7 (muy de acuerdo). Investigaciones actuales han confirmado la utilidad de esta escala en la valoración del estrés laboral, así como la presencia de los dos factores mencionados: irritación cognitiva e irritación emocional (Merino et al., 2006; Mohr et al., 2006).

En lo relativo a la pertinencia y validación de la escala para la población estudiada, en estudios previos se han obtenido índices muy adecuados de consistencia interna para ambas subescalas, irritación cognitiva e irritación emocional, con valores que van desde 0.85 a 0.97. Además, se han obtenido datos positivos de validez, en concreto se han encontrado correlaciones significativas entre la irritación y variables como la depresión $(r=0.29, p<0.01)$, la impaciencia $(r=0.7, p<0.001)$ o la incapacidad para relajarse $(r=0.73, p<0.001)$ (Mohr et al., 2006).

Escala Holandesa de Adicción al Trabajo (Dutch Work Addiction Scale) ([DUWAS]; Schaufeli, Taris Eु Bakker, 2006)

Este instrumento consta de 17 ítems cuyo formato de respuesta es tipo Likert con cuatro anclajes que van desde 1 (casi nunca) hasta 4 (casi siempre) y tiene dos subescalas: la primera, trabajar excesi- 
vamente, DU f1 E, con 9 ítems (ejemplo de ítem: "Me siento culpable cuando no estoy trabajando en algo") y una fiabilidad de 0.67 y la segunda, trabajar de un modo compulsivo, DU f1 C, conformada por 8 ítems (ejemplo de ítem: "Me siento obligado a trabajar duro, incluso cuando no es agradable") y una fiabilidad de 0.77 . Ha sido un instrumento validado con muestras holandesas y españolas (Del Líbano, Llorens, Salanova \& Schaufeli, 2010).

Los indicios de validez de la presente escala se han hallado a través de dos indicadores. En primer lugar, el bienestar psicosocial se evaluó a través de la siguiente pregunta ("En términos generales, ise siente saludable?") en una escala de cuatro anclajes (de 1, casi nunca a 4, casi siempre) la correlación con los dos factores es negativa (DU f1 E; $r=-0.31, p$ $<0.001$ y DU f1 C; $r=-0.28, p<0.001$ ). Por otro lado, la felicidad se evaluó mediante una pregunta ("Teniendo todo en cuenta, iqué tan feliz está con su vida?") a través de una escala de cuatro grados (de 1, infeliz a 4, muy feliz), la asociación hallada fue negativa con los dos factores (DU f1 E; $r=-0.32, p$ $<0.001$ y DU f1 C; $r=-0.31, p<0.001)$.

\section{Procedimiento}

La recogida de datos se llevó a cabo durante un curso escolar. Los integrantes participaron de forma voluntaria y se les informó de las características y objetivos del proyecto. Se llevó a cabo un muestreo aleatorio-accidental (Kerlinger, 2001). La tasa de respuesta fue del $80 \%$. Los participantes contestaron de forma voluntaria, no recibiendo ninguna gratificación ni monetaria ni en especies, en su lugar de trabajo habitual, previo consentimiento de los responsables de las empresas participantes. Se garantizó totalmente la confidencialidad de los datos aportados por los participantes.

\section{Análisis}

La aplicación de los instrumentos de evaluación ha facilitado una información que se ha sometido a diversos análisis estadísticos, utilizando el paquete estadístico SPSS versión 19.0. Por un lado, se han calculado algunos descriptivos y correlaciones; por otro, se han realizado regresiones jerárquicas que han permitido realizar pronósticos y estimaciones entre la adicción al trabajo y la irritación laboral.

\section{Resultados}

En la Tabla 1 aparecen los datos correspondientes a los estadísticos descriptivos, donde se incluyen los datos acerca de la consistencia interna de cada una de las variables, obtenida mediante el coeficiente alpha de Cronbach. Como se puede observar, todas las variables ofrecen unos valores aceptables que van desde 0.67 (DU f1 E) a 0.87 (irritación cognitiva).

A continuación, en la Tabla 2, aparecen los datos correspondientes a las correlaciones entre las variables consideradas en este estudio.

Como se puede observar en la Tabla 2, todas las variables correlacionaron entre sí de forma significativa, a excepción del WBAT f2, que no mostró correlación con ninguno de los componentes de la

TABLA 1

Estadísticos descriptivos

\begin{tabular}{lcccccc}
\hline \multicolumn{1}{c}{ Variables } & $N$ & Mínimo & Máximo & $M$ & $D E$ & $\alpha$ \\
\hline Irritación Emocional & 192 & 5 & 29 & 13.58 & 5.89 & 0.77 \\
Irritación Cognitiva & 194 & 3 & 21 & 9.07 & 5.06 & 0.87 \\
WBAT f1 & 278 & 15 & 56 & 32.67 & 8.86 & 0.82 \\
WBAT f2 & 278 & 7 & 33 & 16.21 & 5.43 & 0.83 \\
DU f1 E & 281 & 6 & 23 & 14.23 & 3.86 & 0.67 \\
DU f1 C & 280 & 4 & 16 & 8.6 & 2.84 & 0.77 \\
\hline
\end{tabular}

Fuente: elaboración propia 
TABLA 2

Matriz de correlaciones Irritación-Adicción al Trabajo

\begin{tabular}{lcccccc}
\hline \multicolumn{1}{c}{ Variables } & 1 & 2 & 3 & 4 & 5 & 6 \\
\hline 1 Irritac. Emocional & - & & & & & \\
2 Irritac. Cognitiva & $0.38^{* *}$ & - & & & & \\
3 WBAT f1 & $0.32^{* *}$ & $0.49^{* *}$ & - & & & \\
4 WBAT f2 & -0.05 & 0.05 & $0.29 * *$ & - & - & \\
5 DU f1 E & $0.26^{* *}$ & $0.49^{* *}$ & $0.62 * *$ & $0.21^{* * *}$ & - & \\
6 DU f1 C & $0.27^{* *}$ & $0.33^{* *}$ & $0.73^{* *}$ & $0.22^{* *}$ & $0.59^{* *}$ & - \\
\hline
\end{tabular}

$* * p<0.01$

Fuente: elaboración propia

irritación laboral, aunque sí con las dos dimensiones del DUWAS.

Por tanto, estos resultados confirman la presencia de una relación positiva y significativa entre los dos componentes de la irritación, la irritación cognitiva y la emocional, y la adicción al trabajo, concretamente con el Factor 1 del WBAT, denominado motivación y también con los dos factores del DUWAS: trabajar de un modo excesivo y trabajar de un modo compulsivo. En el análisis de correlaciones bivariadas, se observa que la irritación cognitiva muestra los valores más altos, por lo tanto será esta la dimensión que se utilizará para el análisis de regresión. Con la irritación emocional, el análisis de regresión arrojó un resultado muy pobre incorporando al modelo sólo al WBAT f1 y explicando muy poca varianza, en torno a un $10 \%$, por lo que se descarta su explicación en este artículo.

Por tanto, a continuación se pasa a ver los resultados que arroja el análisis de regresión, utilizando como variables independientes los dos componentes de la irritación del WBAT y los dos del DUWAS y como variable dependiente la irritación cognitiva.

\section{Ausencia de colinealidad entre las variables independientes}

El índice de tolerancia entre las dos variables independientes, WBAT y DUWAS, alcanzó un valor de 0.611 (FIV $=1.635$ ), por tanto no hay colinealidad, si bien en la tabla de correlaciones anterior se observa una relación significativa entre todas las dimensiones de la adicción $(p<0.01)$. El dato correspondiente a la colinealidad aparece más ade- lante en la Tabla 3. Por tanto, en esta primera fase, se puede concluir que se comprueban los supuestos previos de linealidad y ausencia de colinealidad. Todos ellos se cumplen satisfactoriamente. El diagrama de dispersión y la prueba de significación del coeficiente de Pearson entre irritación cognitiva y WBAT f1 revelan la existencia de una relación directa y altamente significativa con $p<0.05(r=$ $0.49 ; p=0.001$ ). Lo mismo ocurre con el estudio de la relación entre irritación cognitiva y los factores del DUWAS $(r=0.49 ; p=0.001$ y $r=0.33 ; p=$ 0.001). Como conclusión, es posible admitir que es factible construir un modelo de regresión lineal múltiple para predecir irritación desde la adicción al trabajo. En cuanto al índice Durbin-Watson, este alcanzó un valor de 1.938, encontrándose entre los márgenes de referencia 1.5-2.5, lo cual indica que no existe interdependencia entre los residuos.

\section{ANOVA de regresión}

A continuación, en la Tabla 3, aparece el resumen del modelo.

En primer lugar, se puede señalar que se han dado 2 pasos. Como había 4 variables independientes, eso quiere decir que se han incluido 2. Primero, se introduce el DU.f1.E "trabajar excesivamente" (la $r$ de Pearson era ligeramente mayor). Este primer modelo (que sería de regresión simple, al tener solo 1 predictor) tiene un $R^{2}$ del $24.7 \%$, que es bajo pero significativo $(p=0.001)$ en el ANOVA de este primer paso. En la tabla de variables excluidas de este modelo 1, aparecen las otras VI, los dos factores del WBAT y el segundo factor del DUWAS, y 
TABLA 3

Resumen del modeloc

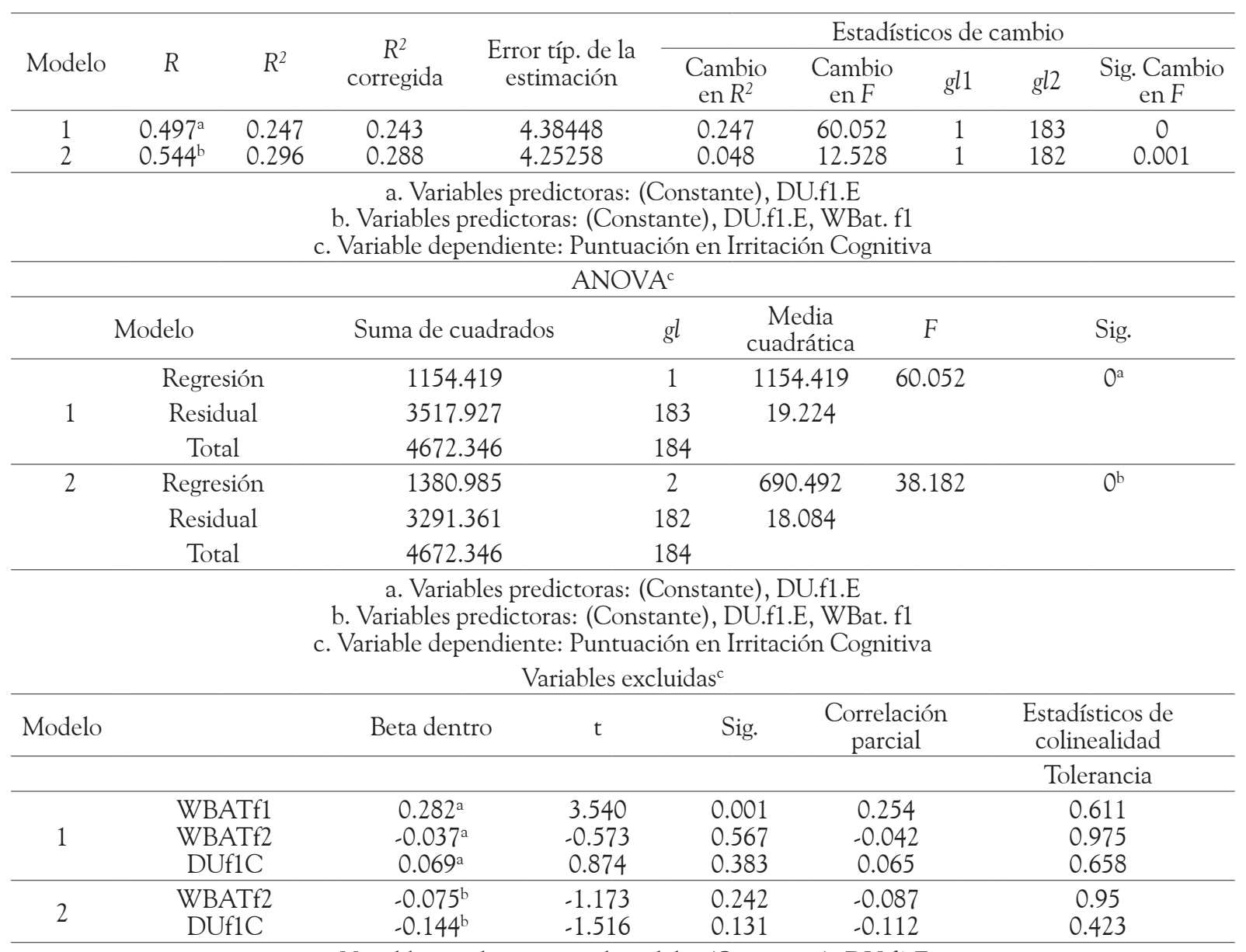

a. Variables predictoras en el modelo: (Constante), DU.f1.E

b. Variables predictoras en el modelo: (Constante), DU.f1.E, WBAT f1

c. Variable dependiente: Puntuación en Irritación Cognitiva

Fuente: elaboración propia

es ahí donde se ve el valor del índice de tolerancia, pero también se ve que, como WBAT f1 "disfrute con el trabajo" tiene $p<0.05$, va a entrar en el segundo paso.

Efectivamente, donde dice modelo 2, se añade WBAT f1 y el valor del $R^{2}$ (conjunto entre ambas) aumenta al 29.6\%; es decir, que esta segunda VI añade un $5 \%$, un valor que sigue siendo bajo. El incremento es significativo, eso es lo que se ve donde dice significación del cambio, $\operatorname{con} p=0.001<0.05$. Y este segundo modelo, que ya es múltiple, también es significativamente eficaz en la reducción de los errores (ANOVA de regresión: $p=0$ ).
En resumen, este modelo múltiple, contiene a las 2 VI predictoras, es significativamente eficaz (la $p=0$ del ANOVA), pero tiene un valor de $R^{2}$ moderado $(29.6 \%)$ por lo que la magnitud de los potenciales errores de pronóstico será alta (el valor del error típico de estimación es 4.25).

\section{Pruebas $\mathrm{T}$ sobre los coeficientes $B$}

La Tabla 4 de coeficientes indica por un lado los valores de las $\mathrm{B}$ con las que se va a construir la ecuación (que esto es la fase tres, en realidad), pero también su significación que es importante en esta fase 2 de evaluación del modelo, puesto que dice 
qué coeficientes B forman parte de la ecuación final. Por coherencia con lo anterior, las B de ambas VI tienen que ser significativas, es justamente lo que se aprecia en la columna "Sig.", en las casillas del modelo 2 (DU.f1.E $p=0.001$ y WBAT.f1 $p=$ 0.001). Lo que queda por ver es si la $B_{0}$ (la constante poblacional) también lo es y su valor forma parte de la ecuación. Junto a las $p$ anteriores se ve la $p$ de esta constante poblacional, es $p=0.279$, por tanto no es significativa y no estará en la ecuación.

\section{Construcción del modelo}

En la misma Tabla 4, vemos los valores de las B en puntuaciones directas (columna de coeficientes no estandarizados) y en la escala de puntuaciones típicas Z. La B de DU.f1.E es 0.406 y eso quiere decir que por cada unidad de medida (1 unidad) que aumenta esta VI, la irritación cognitiva aumenta en algo más de 0.4 puntos. La B de WBAT es 0.152 por lo que por cada $1 \mathrm{um}$ de aumento de esta VI, la VD aumenta en 0.2 puntos. En los resultados se incluyen sus respectivos intervalos de confianza al 95\%. De manera que, la ecuación del modelo de Regresión Lineal Múltiple (RLM) final, incluyendo el error típico, es la siguiente:

Irritación cognitiva $=0.41 \times$ DU.f1.E + 0.15×WBAT.f1 $( \pm 1.96 \times 4.25)$

Todo lo anterior se resume en lo siguiente: El modelo se ha construido en 2 pasos. En el primero de ellos se ha introducido como mejor predictor a DU.f1.E y en el segundo se ha añadido a WBAT.f1. El modelo de RLM conjunto es significativamente eficaz en la reducción de los errores de pronóstico $\operatorname{con} p<0.05$ (ANOVA de regresión: $F=38.182$; 2 y $182 \mathrm{gl} ; p=0$ ). Con todo ello, el modelo alcanza un moderado porcentaje de variabilidad explicada, ligeramente superior a la cuarta parte (coeficiente de determinación: 29.6\%; error típico de estimación: 4.25).

La variable DU.f1.E tiene un peso predictivo de 0.41 um de Irritación cognitiva por cada um ( $p$ $=0 ; \mathrm{IC}$ al 95\%: 0.208 a 0.604). La capacidad de pronóstico de WBAT.f1 es de 0.15 um por cada um $(p=0.001$; IC al 95\%: 0.067 a 0.237).

\section{Discusión}

Este estudio aporta nuevos e interesantes datos a la investigación sobre la irritación laboral y la adicción al trabajo, dos variables muy importantes y que hasta la fecha no habían sido estudiadas de un modo simultáneo. Una vez analizados los resultados, se puede concluir que existe una relación positiva y significativa entre la irritación laboral y la adicción al trabajo, tal como predice el modelo teórico. Haciendo un análisis más pormenorizado, los resultados obtenidos arrojan una relación positiva y significativa entre la irritación laboral, tanto emocional como cognitiva, y los dos factores del DUWAS, trabajar de forma excesiva y trabajar de forma compulsiva, añadiendo evidencia empírica,

\section{TABLA 4}

Coeficientes $^{a}$

\begin{tabular}{|c|c|c|c|c|c|c|c|c|}
\hline & \multirow[t]{2}{*}{ Modelo } & \multicolumn{2}{|c|}{$\begin{array}{l}\text { Coeficientes no } \\
\text { estandarizados }\end{array}$} & \multirow{2}{*}{$\begin{array}{c}\text { Coeficientes } \\
\text { tipificados }\end{array}$} & \multirow[t]{2}{*}{$\mathrm{t}$} & \multirow{2}{*}{ Sig. } & \multicolumn{2}{|c|}{$\begin{array}{l}\text { Intervalo de confianza de } 95 \% \\
\text { para B }\end{array}$} \\
\hline & & $\mathrm{B}$ & Error típ. & & & & Límite inferior & Límite superior \\
\hline \multirow{2}{*}{1} & (Constante) & 0.482 & 1.153 & & 0.418 & 0.676 & -1.793 & 2.757 \\
\hline & DU.f1.E & 0.627 & 0.081 & 0.497 & 7.749 & 0 & 0.468 & 0.787 \\
\hline \multirow{3}{*}{2} & (Constante) & -1.336 & 1.23 & & -1.086 & 0.279 & -3.764 & 1.092 \\
\hline & DU.f1.E & 0.406 & 0.1 & 0.322 & 4.041 & 0 & 0.208 & 0.604 \\
\hline & WBat.f1 & 0.152 & 0.043 & 0.282 & 3.54 & 0.001 & 0.067 & 0.237 \\
\hline
\end{tabular}

a. Variable dependiente: Irritación Cognitiva.

Fuente: elaboración propia 
de este modo, al aspecto negativo de la adicción al trabajo que se anotó en la introducción (Bakker et al., 2013; Killinger, 1991; Porter, 1996; Schaef \& Fassel, 1988). Por otro lado, en el caso del WBAT, solo se han obtenido correlaciones positivas con el Factor 1, el correspondiente a la motivación. Estos resultados son congruentes con el modelo teórico, ya que es más lógico que la irritación laboral correlacione con el componente relativo a motivación que con el componente correspondiente a disfrute, aunque, tal como se señaló en la introducción, la adicción al trabajo también puede tener una visión positiva (Machlowitz, 1980; Naughton, 1987; Sprankle \& Ebel, 1987).

De este estudio, basado en el análisis de regresión, se concluye que la adicción al trabajo está más vinculada a la irritación cognitiva que a la irritación emocional, ya que ésta no se ha incorporado como variable dependiente al modelo planteado. En concreto, la irritación cognitiva es explicada de un modo moderado por el Factor 1 del DUWAS, trabajar de forma excesiva, y por el Factor 1 del WBAT, motivación, tal como era de esperar según el modelo teórico. Efectivamente, tal como se define de forma teórica la irritación cognitiva, es posible esperar que esté asociada a conductas de trabajar de forma excesiva, algo que añade datos importantes al entendimiento de la irritación cognitiva, cuya investigación se ha centrado sobre todo en la consideración y explicación de aspectos cognitivos como, por ejemplo, el pensar de forma recurrente sobre las mismas ideas o las dificultades encontradas en el mundo laboral para conseguir determinados objetivos.

En el modelo de regresión planteado, se observa que la primera dimensión del DUWAS, el DU f1 E, trabajar excesivamente explica un $24.7 \%$ de la irritación cognitiva. Mientras que con la incorporación al modelo del primer factor del WBAT f1, motivación, el porcentaje asciende a un 29.6\%. En resumen, las variables trabajar excesivamente y motivación explican aproximadamente un 30\% de la irritación cognitiva.

Los resultados obtenidos en este estudio permiten reflexionar sobre las implicaciones teóricas, metodológicas o prácticas de los hallazgos y sus repercusiones sobre el modelo generado. Tal como se desprende de los resultados, la irritación laboral se relaciona de un modo significativo con las dimensiones de la adicción al trabajo, excepto con la dimensión positiva como es el disfrute, algo esperado desde el modelo teórico y que refuerza, por tanto, las implicaciones teóricas sobre el modelo explicativo de la adicción al trabajo.

El comportamiento diferente observado entre irritación cognitiva y emocional también permite hacer reflexiones interesantes para explicar el modelo generado. Tal como se veía en el apartado de resultados, la irritación cognitiva muestra correlaciones más altas que la irritación emocional con la adicción al trabajo, concretamente con el factor denominado motivación y también con los dos factores denominados trabajar de un modo excesivo y trabajar de un modo compulsivo. Una posible explicación de este resultado puede ser la propia naturaleza de la irritación cognitiva; hay que tener en cuenta que este tipo de irritación, a diferencia de la irritación emocional, está vinculada a los esfuerzos personales realizados para conseguir objetivos dentro del mundo laboral y puede llevar al empleado a dispensar una mayor dedicación en todo lo que hace. Pero no hay que olvidar que esta irritación cognitiva, si no se regula de un modo adecuado, puede tener efectos negativos y deteriorar el funcionamiento cognitivo del individuo a medio y largo plazo.

De los resultados encontrados en este estudio, también se derivan líneas de intervención en el mundo del trabajo que ya han sido apuntadas por otros autores (Ng et al., 2007). En este apartado simplemente se suscriben y se adaptan a los matices de este estudio y a un entorno de lengua española.

El hecho de que se estén desvaneciendo las fronteras entre la vida laboral, familiar y social, y se adquieran otro tipo de roles donde las fronteras no están claras, puede crear un ambiente favorable para los adictos al trabajo. En esta situación se corre el riesgo de que aumente la irritación cognitiva y se anule la capacidad de desconexión por parte del empleado, esto último deja abierta una interesante línea de investigación para el futuro, es decir, en qué medida un desvanecimiento de fronteras entre vida 
laboral y familiar crea mayor adicción al trabajo y por extensión una mayor irritación cognitiva.

Dado que en la actualidad se espera de los empleados una mayor implicación y responsabilidad en el propio desarrollo de la carrera, se puede crear en los adictos al trabajo un mayor estado de irritación que a largo plazo repercuta de forma negativa en su salud laboral. Por tanto, lo que a corto plazo puede parecer una solución para la organización, a largo plazo puede resultar un problema.

En cuanto a las líneas de intervención a nivel organizacional, aparecen sugerencias importantes para los directivos, ya que por un lado tienen que buscar en los empleados un nivel óptimo de compromiso y, por otro lado, tienen que ser conscientes de que pueden ejercer un modelado negativo al ser ellos mismos adictos al trabajo (Ng et al., 2007). Por esto, los directivos de las organizaciones modernas tienen ante sí un gran desafío para desarrollar con los empleados estilos de gestión que combinen de forma eficiente la motivación y el trabajo audaz, con medidas que permitan a los empleados crear espacios donde puedan desconectar de modo que su nivel de irritación cognitiva no supere ciertos umbrales de riesgo y no les lleve a estados de irritación emocional o situaciones que deterioren su bienestar y la calidad de sus relaciones interpersonales.

En cuanto a las limitaciones del estudio, se pueden señalar las propiamente derivadas de la utilización de técnicas de autoinfome. Además, el tamaño de la muestra utilizada es algo pequeño para tratarse de una muestra multiocupacional, por lo que en el futuro podría incrementarse el número de sujetos o realizar estudios con muestras de profesiones más homogéneas y confirmar, por ejemplo, el comportamiento diferencial que ofrece el WBAT f2, disfrute con el trabajo. Además, deberían incluirse las evaluaciones que los compañeros de trabajo y/o los supervisores (Andreassen, Hetland \& Pallesen, 2010) pueden realizar sobre un empleado. También deberían utilizarse estudios longitudinales, con el fin de determinar las consecuencias de la adicción al trabajo y, además, la relación entre ésta y otras variables como la formación, el crecimiento personal, la promoción profesional y los conflictos trabajo vs. familia (Huang, $\mathrm{Hu} \& \mathrm{Wu}, 2010$ ).
En cuanto a las líneas de investigación futuras, se podrían incorporar variables de personalidad a la relación con la adicción al trabajo, tal como se viene planteando en algunos estudios (Burke et al., 2006; Clark, Lelchook \& Taylor, 2010), por ejemplo, variables contempladas dentro del modelo Big Five, la autoeficacia, la autorregulación, el perfeccionismo, las disposiciones afectivas o el propio compromiso o engagement que puede ejercer un efecto mediador importante en la dedicación al trabajo de un modo idóneo. Otra línea de investigación empírica sería escudriñar la adicción al trabajo y el género, ya que ha sido escasa (Aziz \& Zickar, 2006). Por tanto, deberá investigarse la importancia de los roles de género en la formación de conductas adictivas al trabajo y la posibilidad de distinguir las diferencias de género. Finalmente, se requerirán investigaciones adicionales para examinar el proceso evolutivo por las cuales un adicto al trabajo puede ir transitando en el proceso de adicción (Chamberlin \& Zhang, 2009); es más, sería necesario identificar cómo la adicción al trabajo afecta a las personas en las diferentes etapas que configuran su ciclo vital y profesional.

\section{Referencias}

Andreassen, C. S., Hetland, J., \& Pallesen, S. (2010). The relationship between 'workaholism', basic needs, satisfaction at work and personality. European Journal of Personality, 24(1), 3-17.

Aziz, S., \& Zickar, M. J. (2006). A cluster analysis investigation of workaholism as a syndrome. Journal of Occupational Health Psychology, 11(1), 52-62.

Bakker, A. B., Demerouti, E., Oerlemans, E., \& Sonnentag, S. (2013). Workaholism and daily recovery: A day reconstruction study of leisure activities. Journal of Organizational Behavior, 34(1), 87-107.

Balducci, C., Cecchin, M., Fraccaroli, F., \& Schaufeli, W. B. (2012). Exploring the relationship between workaholism and workplace aggressive behaviour: The role of job-related emotion. Personality and Individual Differences, 53(5), 629-634.

Berglas, S. (2004). Treating workaholism. En R. H. Coombs (Ed.), Handbook of addictive disorders (pp. 383-407). New Jersey: John Wiley and Sons, Inc. 
Boada-Grau, J., Prizmic-Kuzmica, A. J., Serrano-Fernández, M. J. \& Vigil-Colet, A. (2013). Estructura factorial, fiabilidad y validez de la escala de adicción al trabajo (WorkBAT): Versión española. Anales de Psicología, 29(3), 923-933.

Burke, R. J. (1999). Workaholism in organizations. Measurement validation and replication. International Journal of Stress Management, 6, 45-55.

Burke, R. J. (2001). Workaholism components, job satisfaction and career progress. Journal of Applied Social Psychology, 30, 637-645.

Burke, R. J., Matthiesen, S. B., \& Pallesen, S. (2006). Personality correlates of workaholism. Personality and Individual Differences, 40(6), 1223-1233.

Chamberlin, C. M., \& Zhang, N. (2009). Workaholism, health, and self-acceptance. Journal of Counseling E Development, 87(2), 159-169.

Cherrington, D. J. (1980). The work ethic. New York: American Management Association.

Clark, M. A., Lelchook, A. M., \& Taylor, M. L. (2010). Beyond the Big Five: How narcissim, perfectionism, and dispositional affect relate to workaholism. Personality and Individual Differences, 48(7), 786-791.

Cooper, G. L. (1998). Controversy and contentions: The changing nature of work. Community, Work, and Family, 1(3), 313-317.

Del Líbano, M., Llorens, S., Salanova, M., \& Schaufeli, W. (2010). Validity of a brief workaholism scale. Psicothema, 22(1), 143-150.

Fassel, D. (1990). Working ourselves to death: The high costs of workaholism, the rewards of recovery. San Francisco: Harper Collins.

Fletcher, J. K., \& Baylin, L. (1996). Challenging the last boundary: Reconecting work and family. En M. B. Arthur \& D. M. Rousseau (Eds.), The boundaryless career (pp. 256-267). New York: Oxford University Press.

Huang, J. -C., Hu, C., \& Wu, T. -C. (2010). Psychometric properties of the Chinese version of the workaholism battery. The Journal of Psychology, 144(2), 163-183.

Kanai, A., Wakabayashi, M., \& Fling, S. (1996). Workaholism among employees in Japanese corporations: An examination based on the Japanese version of the workaholism scales. Japanese Psychological Research, 38(4), 192-203.

Kerlinger, F. N. (2001). Investigación del comportamiento: métodos de investigación en ciencias sociales. México: McGraw-Hill.

Killinger, B. (1991). Workaholics: The respectable addicts. New York: Simon \& Schuster.

Locke, E. A., \& Latham, G. P. (1990). A theory of goal setting and task performance. Englewood Cliffs, NJ: Prentice-Hall.

Machlowitz, M. (1980). Workaholics: Living with them, working with them. Reading, MA: Addison-Wesley.

McMillan, L. H. W., Brady, E. C., O'Driscoll, M. P., \& Marsh, N. V. (2002). A multifaceted validation study of Spence and Robbins' (1992) workaholism battery. Journal of Occupational and Organizational Psychology, 75(3), 357-368.

Merino, E., Carbonero, M. A., Moreno, B., \& Morante, M. E. (2006). La Escala de Irritación como instrumento de evaluación del estrés laboral. Psicothema, 18(3), 419-424.

Mohr, G. (1986). Die Erfassung Psychischer Befindensbeeinträchtigungen bei Industriearbeitern. Europäische Hochschulschriften [The assessment of mental strain of industrial workers]. Frankfurt: Lang.

Mohr, G., Müller, A., Rigotti, T., Aycan, Z., \& Tschan, F. (2006). The assessment of psychological strain in work contexts: Concerning the structural equivalency of nine language adaptations of the Irritation Scale. European Journal of Psychological Assessment, 22(3), 198-206.

Naughton, T. J. (1987). A conceptual view of workaholism and implications for career counselling and research. Career Development Quarterly, 58(2), 367-408.

Ng, T. W. H., Sorensen, K. L., \& Feldman, D. C. (2007). Dimensions, antecedents, and consequences of workaholism: A conceptual integration and extension. Journal of Organizational Behavior, 28(1), 111-136. doi: 10.1002/job.424

Oates, W. (1971). Confessions of a workaholic. New York: World.

Porter, G. (1996). Organizational impact of workaholism: Suggestions for researching the negative outcomes of excessive work. Journal of Occupational Health Psychology, 1(1), 70-84. 
Robinson, B. E. (1998). Chained to the desk: A guidebook for workaholic, their partners and children and clinicians who treat them. New York: NYU Press.

Salanova, M., Schaufeli, W. B., Llorens, S., Peiró, J. M., \& Grau, R. (2000). Desde el 'burnout' al 'engagement': iuna nueva perspectiva? Revista de Psicología del Trabajo y las Organizaciones, 16(2), 117-134.

Schaef, A. W., \& Fassel, D. (1988). The addictive organization. San Francisco, CA: Harper \& Row.

Schaufeli, W. B., Taris, T. W., \& Bakker, A. (2006). Dr. Jekyll and Mr. Hide: On the differences between work engagement and workaholism. En R. Burke (Ed.), Research companion to working time and work addiction (pp. 193-217). Northhampton, MA: Edward Elgar.

Scott, K. S., Moore, K. S., \& Miceli, M. P. (1997). An exploration of the meaning and consequences of workaholism. Human Relations, 50(3), 287-314.
Smith, D. E., \& Seymour, R. B. (2004). The nature of addiction. En R. H. Coombs (Ed.), Handbook of addictive disorders: A practical guide to diagnosis and treatment (pp. 3-30). New Jersey: John Wiley $\&$ Sons, Inc.

Spence, J. T., \& Robbins, A. S. (1992). Workaholism: Definition, measurement, and preliminary results. Journal of Personality Assessment, 58(1), 160-178.

Sprankle, J. K., \& Ebel, H. (1987). The workaholic syndrome. New York: Walker.

Sullivan, S. E. (1999). The changing nature of careers. A review and research agenda. Journal of Management, 25(3), 457-484.

Van Beek, I., Taris, T. W., \& Schaufeli, W. (2011). Workaholic and work engaged employees: Dead ringers or world apart? Journal of Occupational Health Psychology, 16(4), 468-482. 
